MPI-PhT/95-97

LMU $18 / 95$

hep-ph/9510294

October 1995

\title{
B Meson Form Factors and Exclusive Decays ${ }^{1, *}$
}

\author{
A. Khodjamirian ${ }^{a, \dagger}$ and R. Rückl ${ }^{a, b}$ \\ ${ }^{a}$ Sektion Physik der Universität München, D-80333 München, Germany \\ ${ }^{b}$ Max-Planck-Institut für Physik, Werner-Heisenberg-Institut, D-80805 München, Germany
}

\begin{abstract}
We present results for the $B \rightarrow \pi$ and $D \rightarrow \pi$ form factors derived from QCD sum rules on the light-cone. Our predictions are compared with experiment and used to extract the quark mixing parameter $\left|V_{u b}\right|$ from a recent CLEO measurement of $B \rightarrow$ $\pi l^{+} \nu$. Furthermore, we discuss the factorization approximation for exclusive nonleptonic matrix elements, and describe a first QCD estimate of the nonfactorizable contribution to the amplitude of $B \rightarrow J / \psi K$.
\end{abstract}

${ }^{\dagger}$ on leave from Yerevan Physics Institute, 375036 Yerevan, Armenia

${ }^{1}$ supported by the German Federal Ministry BMBF under contract 05 6MU93P, and by the EC-program HCM under contract CHRX-CT93-0132

*invited talk presented by R. Rückl at the Workshop 'Beauty 95', Oxford, July 9-14,1995, to appear in the proceedings 


\section{Introduction}

Heavy flavour decays play an outstanding role in the determination of fundamental parameters of the standard model and for the development of a deeper understanding of the QCD dynamics. In order to achieve the ambitious scientific goals it is important to combine the experimental and theoretical efforts. However, this is not always easy. For example, while the theory of inclusive decays is most advanced, inclusive measurements are generally quite difficult. Conversely, exclusive decays into few-body final states are often much easier to detect and reconstruct, but the theoretical methods to treat exclusive processes are still rudimentary when hadrons are involved. In view of the exciting experimental prospects at future bottom and charm factories, where many new exclusive channels will become accessible, it appears particularly desirable to have a reliable quantitative theory.

As well known, the main problem is the complicated interplay of weak and strong interactions, and most of all the influence of long-distance dynamics. Clearly, a complete theoretical understanding of the decays of hadrons containing a heavy quark is only possible in a nonperturbative framework. This is most obvious for exclusive nonleptonic decays and for processes involving strong couplings among hadrons such as $B^{*} B \pi$ or $D^{*} D \pi$. Yet, what appears as a problem for determining electroweak parameters from exclusive measurements can also be regarded as a chance to improve and test our understanding of hadronic physics in QCD.

The nonperturbative approaches applied in this field include lattice approximations, heavy quark effective theory, chiral perturbation theory, $1 / N_{c}$-expansion, QCD sum rules, and quark models. Each of these methods has advantages and disadvantages. For example, quark models are easy to use and very good for intuition, however, their relation to QCD is unclear. In the other extreme, lattice calculations are rigorous from the point of view of QCD, but they can (at least presently) be applied only to relatively simple matrix elements related to decay constants and form factors. Moreover, there are uncertainties connected with the necessary extrapolations to the physical masses of the light $(\mathrm{u}, \mathrm{d})$ and heavy $(\mathrm{b})$ quarks. Also the application of effective theories is restricted to a rather narrow class of problems, and the corrections are often substantial. More importantly, in many cases the latter cannot be calculated directly within the same theory, and therefore bring in model-dependence after all.

QCD sum rules [1] represent no exception is this respect. On the one hand, they are based on first principles. On the other hand, one has to introduce new elements such as vacuum condensates or light-cone wave functions which cannot (yet) be calculated directly in QCD. However, since this nonperturbative input is of universal nature, sum rules nevertheless possess a high predictive power. Furthermore, the sum rule approach is particularly well suited for heavy quark physics, since the approximations which have to be made in practice can be well justified when a heavy mass scale is present. Last but not least, the sum rule approach is less limited in applicability than some of the other methods mentioned, or the limitations are different. In this paper we will exemplify some recent applications of the QCD sum rule technique to $B$ and, for comparison, also to $D$ decays.

The simplest and therefore most instructive example for introduction is actually provided by the calculation of heavy meson decay constants. They are defined by matrix elements of quark currents, for example

$$
m_{b}\left\langle B\left|\bar{b} i \gamma_{5} d\right| 0\right\rangle=f_{B} m_{B}^{2},
$$

and determine leptonic decays such as $B \rightarrow \tau \nu_{\tau}$. In addition they enter in the factorizable amplitudes of nonleptonic decays. Without going into details, which for example can be found in [2], we only mention that the QCD sum rule approach to (1]) is based on an analysis of 
the two-point correlation function $\left\langle 0\left|T\left\{\bar{b}(x) i \gamma_{5} d(x), \bar{d}(0) i \gamma_{5} b(0)\right\}\right| 0\right\rangle$ using operator product expansion, perturbation theory, and dispersion relations. $T$ in the above current-correlator denotes the time-ordering operation.

For brevity, we directly proceed to more complicated cases and begin with the calculation of form factors. In section 2, we describe how sum rules on the light-cone can be used to derive the $B \rightarrow \pi$ and $D \rightarrow \pi$ form factors. Comparison with recent experimental results on CKM-suppressed $B$ and $D$ decays as well as the determination of $\left|V_{u b}\right|$ are discussed in section 3. Finally, section 4 is devoted to the main theoretical problem in heavy quark decays that is the complete calculation of exclusive nonleptonic amplitudes. We briefly demonstrate the failure of strict factorization, the usual phenomenological procedure, and a first attempt to estimate the nonfactorizable piece of the amplitude for $B \rightarrow J / \psi K$.

\section{$2 \quad$ The $B \rightarrow \pi$ and $D \rightarrow \pi$ form factors}

Recently, the CLEO collaboration [3] announced the first measurement of the decay $B \rightarrow \pi l \nu$. This process plays a very important role for the determination of the CKM parameter $V_{u b}$. The decay amplitude is completely determined by the form factor $f_{B}^{+}\left(p^{2}\right)$ entering the matrix element

$$
<\pi(q)\left|\bar{u} \gamma_{\mu} b\right| B(p+q)>=2 f_{B}^{+}\left(p^{2}\right) q_{\mu}+\left[f_{B}^{+}\left(p^{2}\right)+f_{B}^{-}\left(p^{2}\right)\right] p_{\mu},
$$

where the assignment of the momenta $p$ and $q$ is quite obvious. In [4 this form factor was calculated from a QCD sum rule on the light-cone. In the following we briefly outline the method.

One starts from the correlation function of the relevant vector and pseudoscalar currents between the vacuum and the on-shell pion state:

$$
\begin{array}{r}
F_{\mu}(p, q)=i \int d^{4} x e^{i p x}\left\langle\pi(q)\left|T\left\{\bar{u}(x) \gamma_{\mu} b(x), \bar{b}(0) i \gamma_{5} d(0)\right\}\right| 0\right\rangle \\
=F\left(p^{2},(p+q)^{2}\right) q_{\mu}+\tilde{F}\left(p^{2},(p+q)^{2}\right) p_{\mu} .
\end{array}
$$

Writing a dispersion relation in the variable $(p+q)^{2}$, one readily finds the $B$ meson ground state contribution to the invariant function $F\left(p^{2},(p+q)^{2}\right)$ by inserting a complete sum of states between the currents in (3), focusing on the term $|B\rangle\langle B|$ and using the relations (11) and (2):

$$
F\left(p^{2},(p+q)^{2}\right)=\frac{2 m_{B}^{2} f_{B} f_{B}^{+}\left(p^{2}\right)}{m_{b}\left(m_{B}^{2}-(p+q)^{2}\right)}+\ldots .
$$

The ellipses in the above denote the contributions from the excited $B$ and from continuum states.

The main idea of the method is to apply the operator product expansion (OPE) to the product of currents in the correlation function (3) at spacelike momentum $(p+q)^{2}<0$ such that the b-quark propagating from point $x$ to point 0 is highly virtual. Moreover, the expansion is performed near the light-cone, $x^{2}=0$, rather than around $x=0$. Consequently, one ends up with a series of bilocal rather than local operators. More definitely, after contracting the $b$ quark fields in (3) and expanding the $b$-quark propagator near $x^{2}=0$, the correlation function can be expressed in terms of quark-antiquark and quark-antiquark-gluon matrix elements of the type

$$
\left\langle\pi(q)\left|\bar{u}(x) \Gamma_{a} d(0)\right| 0\right\rangle \text { and }\left\langle\pi(q)\left|\bar{u}(x) g_{s} G^{\mu \nu}(v x) \Gamma_{b} d(0)\right| 0\right\rangle
$$


respectively, where $\Gamma_{a, b}$ denote certain combinations of Dirac matrices and the auxiliary variable $v$ varies from 0 to 1 . These matrix elements are essentially nonperturbative objects. Most importantly, they are universal and process-independent. In particular, the same matrix elements enter in the corresponding expansion of the correlation function interpolating the $D \rightarrow \pi$ form factor. The latter can be immediately obtained from (3) by replacing $b$ by $c$ and $B$ by $D$.

The vacuum-to-pion matrix elements introduced above can be parameterized in terms of so-called light-cone wave functions with given twist [5, 6, 7]. The most important of them is the leading twist 2 wave function $\varphi_{\pi}(u, \mu)$ defined by

$$
\left\langle\pi(q)\left|\bar{u}(x) \gamma_{\mu} \gamma_{5} d(0)\right| 0\right\rangle=-i q_{\mu} f_{\pi} \int_{0}^{1} d u e^{i u q x} \varphi_{\pi}(u, \mu)+\ldots
$$

where $\mu^{-1}$ is a characteristic distance scale and $u$ is the fraction of the pion light-cone momentum $q_{0}+q_{3}$ carried by the constituent quark. The ellipses in (6) stand for the higher twist components. Asymptotically, that is at $\mu \rightarrow \infty$, QCD perturbation theory implies $\varphi_{\pi}(u, \infty)=6 u(1-u)$. However, at the physical scale $\mu \sim m_{b}$, at which the OPE is applied to the correlation function (3), important nonasymptotic effects are to be expected. These are parameterized by the coefficients $a_{i}(\mu)$ in the following series of Gegenbauer polynomials:

$$
\varphi_{\pi}(u, \mu)=6 u(1-u)\left[1+\sum_{i=2,4, . .} a_{i}(\mu) C_{i}^{3 / 2}(2 u-1)\right] .
$$

Although the input values of the coefficients $a_{i}(\mu)$ at a fixed low momentum scale $\mu$ are unknown, the scale-dependence of $a_{i}(\mu)$ is dictated by the renormalization group. From what is said above, it is also clear that $a_{i}(\mu) \rightarrow 0$ for $\mu \rightarrow \infty$. Over the years a great deal has been learned about these wave functions. They have been classified up to twist 4 and the asymptotic form has been determined. Also various nonasymptotic corrections have been estimated from QCD sum rules for light hadrons. In our calculation we have used the wave functions given in [7].

Substitution of (6) into the light-cone expansion of the correlation function (3) yields a representation of the invariant amplitude $F$ in terms of perturbative and nonperturbative parameters and functions of QCD. The leading term of it reads

$$
F\left(p^{2},(p+q)^{2}\right)=m_{b} f_{\pi} \int_{0}^{1} \frac{d u \varphi_{\pi}(u, \mu)}{m_{b}^{2}-(p+u q)^{2}}+\ldots
$$

Equating now the QCD result (8) in the region of validity at $(p+q)^{2}<0$ with the dispersion relation (4) one obtains a raw sum rule relation for $f_{B} f_{B}^{+}\left(p^{2}\right)$. The rest of the calculation follows the usual QCD sum rule procedure: Borel transformation in $(p+q)^{2}$ and subtraction of the contribution from higher states invoking quark-hadron duality. Details can be found in the original paper [4]. One finally arrives at an expression for the desired form factor, the dominant term of which is given by

$$
f_{B}^{+}\left(p^{2}\right)=\frac{f_{\pi} m_{b}^{2}}{2 f_{B} m_{B}^{2}} \int_{\Delta}^{1} \frac{d u}{u}\left(\varphi_{\pi}\left(u, \mu_{b}\right)+\ldots\right) \exp \left(\frac{m_{B}^{2}}{M^{2}}-\frac{m_{b}^{2}-p^{2}(1-u)}{u M^{2}}\right) .
$$

Here, $M$ is the Borel mass parameter, and the scale $\mu_{b}$ is of order of the characteristic virtuality of the correlation function, $\mu_{b}^{2}=m_{B}^{2}-m_{b}^{2}$. The integration limit $\Delta=\left(m_{b}^{2}-p^{2}\right) /\left(s_{0}-p^{2}\right)$ 
depends on the effective threshold $s_{0}$ above which the contribution from higher states to the dispersion relation (4) are cancelled against the corresponding piece in the QCD representation (8). The twist 3 and 4 contributions denoted by ellipses have also been calculated in [4, 8], but are not shown here for brevity. They involve further quark-antiquark wave functions as well as three-particle quark-antiquark-gluon wave functions.

An important detail which should be mentioned here concerns the treatment of $f_{B}$ in the sum rule (9). For consistency, $f_{B}$ is replaced by the appropriate two-point sum rule, disregarding the $O\left(\alpha_{s}\right)$ gluon corrections since the latter are also not yet included in (9). This procedure considerably decreases the sensitivity of $f_{B}^{+}$to the choice of $m_{b}$ and $s_{0}$ as demonstrated in [4, 8]. Furthermore, we observe a remarkable stability of (9) with respect to a variation of the Borel parameter $M$. The appropriate range of $M^{2}$ is found by requiring the higher twist terms and simultaneously, the contributions from heavier states to remain subleading.

In Fig. 1a the form factor $f_{B}^{+}\left(p^{2}\right)$ is plotted versus $p^{2}$ for the central value $M^{2}=10 \mathrm{GeV}^{2}$ of the fiducial range. On general grounds, one can expect the sum rule (9) to be valid up to a timelike momentum transfer $p^{2}=m_{b}^{2}-O\left(1 \mathrm{GeV}^{2}\right)$. Actually, the calculation shows that above $p^{2} \simeq 17 \div 20 \mathrm{GeV}^{2}$ the stability in $M^{2}$ is lost. In Fig. 2 we compare our prediction with the results obtained in a quark model [9] and from a three-point sum rule involving local quark and gluon condensates instead of light-cone wave functions [10].

Obvious replacements in (3) and (9) yield the corresponding sum rule for the $D \rightarrow \pi$ form factor. The numerical result for $f_{D}^{+}\left(p^{2}\right)$ is plotted in Fig. 1b taking $M^{2}=4 \mathrm{GeV}^{2}$ in accordance with the reliability criteria pointed out above. Note again that this calculation should only be trusted at $p^{2}<m_{c}^{2}-O\left(1 \mathrm{GeV}^{2}\right)$.

For easy comparison with other theoretical results (a rather complete compilation can be found in [11]) we also quote our predictions at $p^{2}=0$ :

$$
f_{B}^{+}(0)=0.29 \pm 0.01 \quad, \quad f_{D}^{+}(0)=0.66 \pm 0.03
$$

The uncertainty above only refers to the variation with $M^{2}$ within the fiducial range. We have also investigated the sensitivity to nonasymptotic effects in the light-cone wave functions. To this end we have recalculated $f_{B}^{+}$and $f_{D}^{+}$using purely asymptotic wave functions and find that the form factors change by less than $10 \%$. This gives additional confidence in the results exhibited in Fig. 1.

For an accurate theoretical analysis of the semileptonic decays $B \rightarrow \pi l \nu$ and $D \rightarrow \pi l \nu$ one also has to know the form factors at larger values of $p^{2}$, ideally up to the zero recoil points $\left(m_{B}-m_{\pi}\right)^{2}$ and $\left(m_{D}-m_{\pi}\right)^{2}$, respectively. At such large $p^{2}$ the form factors are expected to be dominated by the $B^{*}$ and $D^{*}$ poles, respectively. In this approximation,

$$
f_{B}^{+}\left(p^{2}\right)=\frac{f_{B^{*}} g_{B^{*} B \pi}}{2 m_{B^{*}}\left(1-p^{2} / m_{B^{*}}^{2}\right)},
$$

where $g_{B^{*} B \pi}$ is the $B^{*} B \pi$ coupling defined by

$$
\left\langle B^{*-}(p) \pi^{+}(q) \mid \bar{B}^{0}(p+q)\right\rangle=-g_{B^{*} B \pi} q_{\mu} \epsilon^{\mu} .
$$

It is one of the advantages of the light-cone sum rule method that the $B^{*} B \pi$ coupling can be calculated [8] from the same correlation function (3). One only has to set up sum rules with the respect to both the $B$ - and $B^{*}$ - channel considering a dispersion relation in $(p+q)^{2}$ and $p^{2}$, simultaneously. After double Borel transformation of the invariant amplitude (8) involving 
the mass parameters $M_{1}$ and $M_{2}$ one obtains an expression for the product $f_{B} f_{B^{*}} g_{B^{*} B \pi}$, the leading twist term of which depends on the pion wave function $\varphi_{\pi}(u, \mu)$ at the fixed momentum fraction $u=M_{1}^{2} /\left(M_{1}^{2}+M_{2}^{2}\right) \simeq 1 / 2$ :

$$
f_{B} f_{B^{*}} g_{B^{*} B \pi}=\frac{m_{b}^{2} f_{\pi}}{m_{B}^{2} m_{B^{*}}} e^{\frac{m_{B}^{2}+m_{B *}^{2}}{2 M^{2}}}\left\{M^{2}\left[e^{-\frac{m_{b}^{2}}{M^{2}}}-e^{-\frac{s_{0}}{M^{2}}}\right] \varphi_{\pi}(1 / 2)+\ldots\right\}
$$

with $M^{2}=M_{1}^{2} M_{2}^{2} /\left(M_{1}^{2}+M_{2}^{2}\right)$. Taking $\varphi_{\pi}(1 / 2, \mu=0.5 \mathrm{GeV})=1.2 \pm 0.2$ [7] together with the corresponding values of the higher twist wave functions and dividing by the values of $f_{B}$ and $f_{B^{*}}$ as given by the appropriate two-point sum rules, we find, numerically,

$$
g_{B^{*} B \pi}=29 \pm 3
$$

where the indicated error again quantifies the variation within the fiducial range of the Borel parameters.
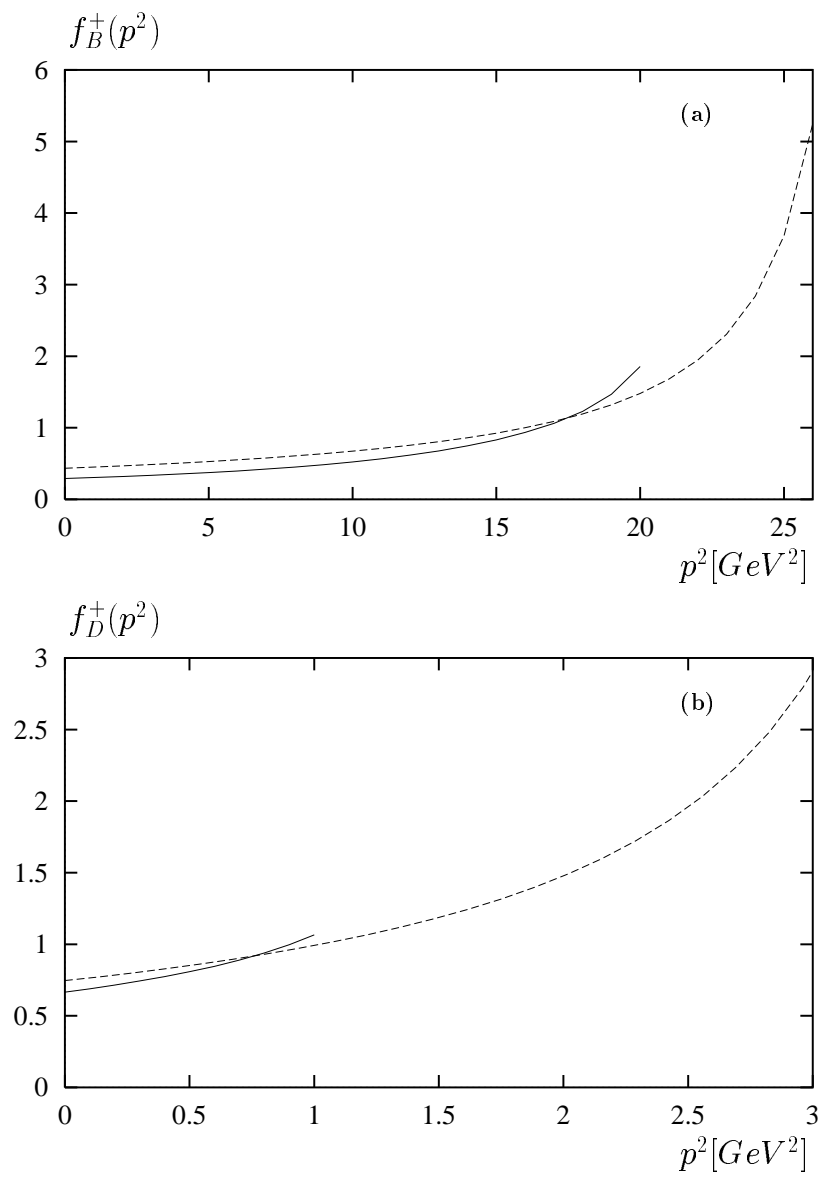

Fig. 1. The form factors for the transitions (a) $B \rightarrow \pi$ and (b) $D \rightarrow \pi$ as predicted by light-cone sum rules (solid) in comparison to the single-pole approximation (dashed) with the normalization constants $g_{B^{*} B \pi}$ and $g_{D^{*} D \pi}$, respectively, calculated by the same method.

Expressions analogous to (11) and (12) hold for $f_{D}^{+}\left(p^{2}\right)$ in the region near $p^{2} \simeq\left(m_{D}-m_{\pi}\right)^{2}$. Following the same procedure as the one described above, we get

$$
g_{D^{*} D \pi}=12.5 \pm 1.0
$$


This prediction implies the partial width

$$
\Gamma\left(D^{*+} \rightarrow D^{0} \pi^{+}\right)=32 \pm 5 \mathrm{keV}
$$

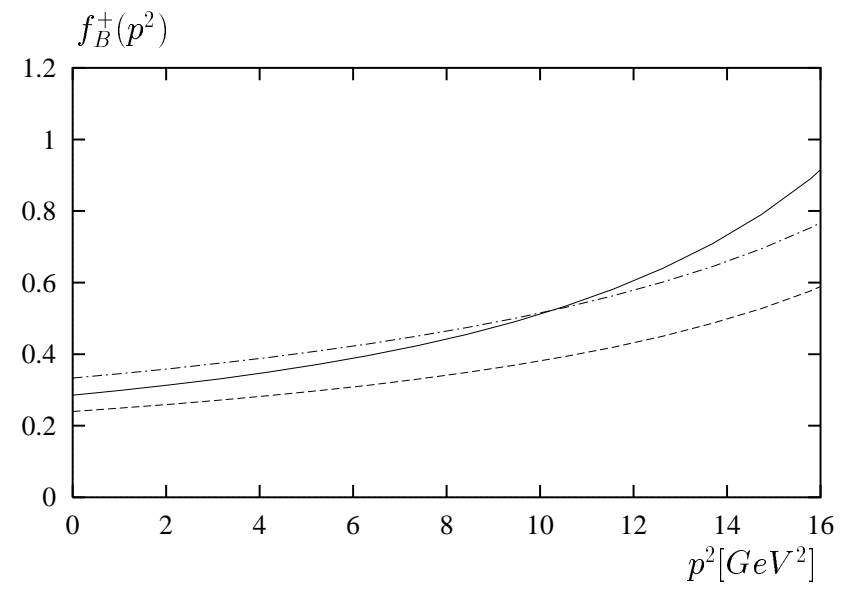

Fig. 2. Comparison of the $B \rightarrow \pi$ form factor as derived from the light-cone sum rule (solid) with the predictions of a three-point sum rule [10] (dashed) and a quark model [9] (dash-dotted).

which is consistent with the limit derived from recent ACCMOR [12] and CLEO [13] measurements.

The single-pole approximation for the form factors $f_{B}^{+}\left(p^{2}\right)$ and $f_{D}^{+}\left(p^{2}\right)$ obtained with (14) and (15) is shown in Fig. 1a and Fig. 1b, respectively. Whereas the direct estimate of the form factors such as (9) is trustful at low and intermediate values of $p^{2}$, but breaks down as $p^{2} \rightarrow m_{b}^{2}$ and $m_{c}^{2}$, respectively, there are no convincing arguments in favour of the pole model to be valid at $p^{2} \rightarrow 0$. Hence we suggest to match the two descriptions in the region of intermediate momentum transfer near the upper end of the full curves plotted in Fig. 1. More precisely, in the following applications we use the direct calculation of $f_{B}^{+}\left(f_{D}^{+}\right)$up to $p^{2}=15(1.0) \mathrm{GeV}^{2}$ and the single-pole approximation at higher values of $p^{2}$.

In order to improve these calculations further one has to take into account the $O\left(\alpha_{s}\right)$ hard gluon exchanges in the correlation function (3) explicitly. Parts of this not quite straightforward task have already been completed [14].

\section{Extraction of $V_{u b}$ from $B \rightarrow \pi l \nu$}

With the form factor $f_{B}^{+}\left(p^{2}\right)$ at hand, we are now in the position to determine the CKM parameter $\left|V_{u b}\right|$ from the recent CLEO measurement of $B^{0} \rightarrow \pi^{-} l^{+} \nu_{l}$. Fig. 3a shows the charged lepton energy spectrum in the $B^{0}$ rest frame,

$$
\begin{array}{r}
\frac{d \Gamma\left(B^{0} \rightarrow \pi^{-} l^{+} \nu_{l}\right)}{d E_{l}}=\frac{G^{2}\left|V_{u b}\right|^{2}}{16 \pi^{3} m_{B}} \int_{0}^{p_{\max }^{2}} d p^{2}\left[2 E_{l}\left(m_{B}^{2}-m_{\pi}^{2}+p^{2}\right)\right. \\
\left.-m_{B}\left(p^{2}+4 E_{l}^{2}\right)\right]\left[f_{B}^{+}\left(p^{2}\right)\right]^{2},
\end{array}
$$


with $p_{\max }^{2}\left(E_{l}\right)=2 E_{l}\left(m_{B}-m_{\pi}^{2} /\left(m_{B}-2 E_{l}\right)\right)$. Integration of the spectrum over $E_{l}$ yields the exclusive decay width

$$
\Gamma\left(B^{0} \rightarrow \pi^{-} l^{+} \nu_{l}\right)=8.1\left|V_{u b}\right|^{2} \mathrm{ps}^{-1}
$$

Experimentally, combining the preliminary CLEO result, $B R\left(B^{0} \rightarrow \pi^{-} l^{+} \nu_{l}\right)=(1.63 \pm$ $0.46 \pm 0.34) \cdot 10^{-4}$ [3], with the world average of the $B^{0}$ lifetime [15], $\tau_{B^{0}}=1.57 \pm 0.05 \mathrm{ps}$, one obtains

$$
\Gamma\left(B^{0} \rightarrow \pi^{-} l^{+} \nu_{l}\right)=(1.04 \pm 0.37) \cdot 10^{-4} \mathrm{ps}^{-1},
$$

where the errors have been added in quadrature. We take the CLEO result obtained by using the BSW model [9] for the efficiency estimate since the BSW form factors agree quite well with our predictions.

Comparison of (18) with (19) yields

$$
\left|V_{u b}\right|=0.0036 \pm 0.0006
$$

The theoretical uncertainty which we estimate conservatively to be less than $20 \%$ is not included in (20).
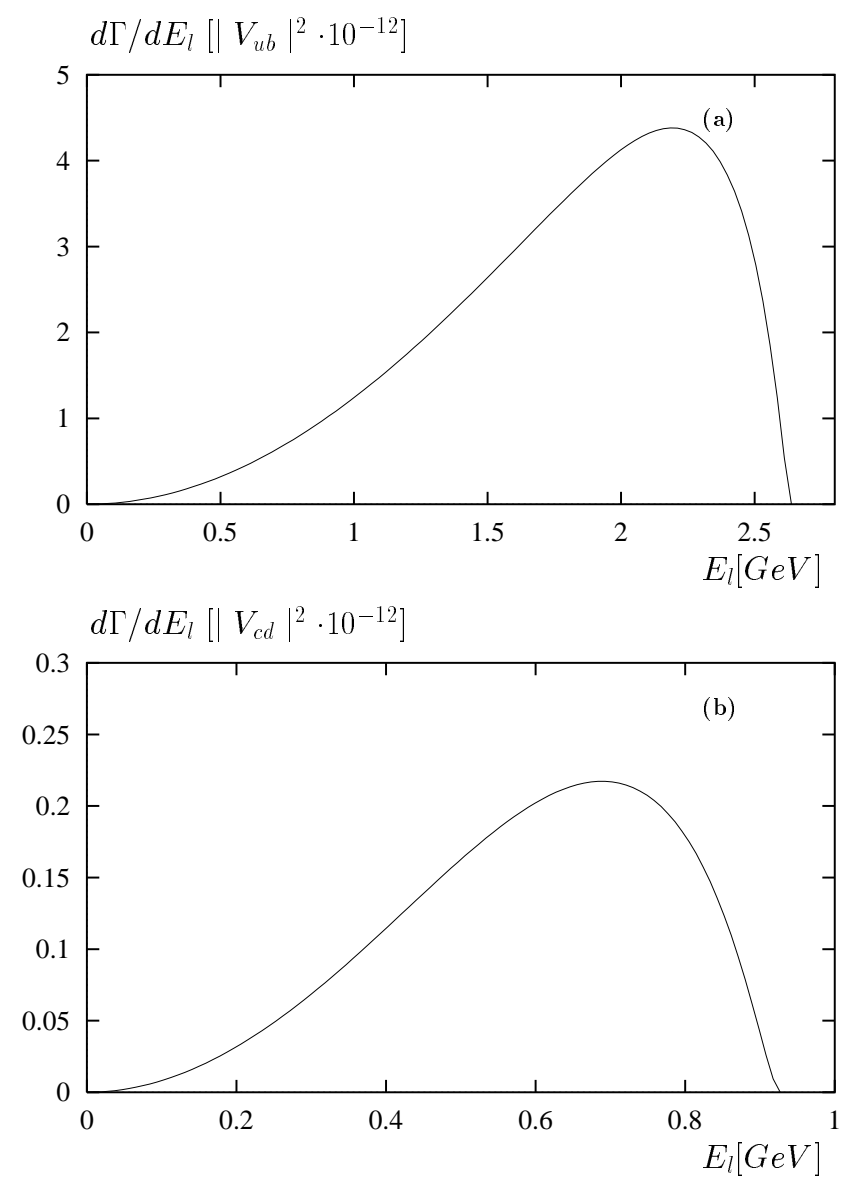
Fig. 3. The charged lepton spectra in (a) $B^{0} \rightarrow \pi^{-} l^{+} \nu$ and (b) $D^{0} \rightarrow \pi^{-} l^{+} \nu$.

In the case of the Cabibbo suppressed $D$ meson decay $D^{0} \rightarrow \pi^{-} l^{+} \nu_{l}$ we predict the charged lepton energy spectrum shown in Fig. 3b and the integrated semileptonic width

$$
\Gamma\left(D^{0} \rightarrow \pi^{-} l^{+} \nu_{l}\right)=0.156\left|V_{c d}\right|^{2} \mathrm{ps}^{-1}=(7.6 \pm 0.2) \cdot 10^{-3} \mathrm{ps}^{-1},
$$

where we have substituted $\left|V_{c d}\right|=0.221 \pm 0.003$ [16].

This prediction should be compared with the experimental result

$$
\Gamma\left(D^{0} \rightarrow \pi^{-} e^{+} \nu_{e}\right)=\left(9.4_{-2.9}^{+5.5}\right) \cdot 10^{-3} \mathrm{ps}^{-1},
$$

derived from the branching ratio $B R\left(D^{0} \rightarrow \pi^{-} e^{+} \nu\right)=\left(3.9_{-1.2}^{+2.3}\right) \cdot 10^{-3}$ and the lifetime $\tau_{D^{0}}=$ $0.415 \pm 0.004 \mathrm{ps}$ [16]. The present theoretical uncertainty in (21) is of the order of the experimental error in (22).

We see that the CKM-suppressed exclusive semileptonic widths are not yet measured precisely enough to really challenge theory. In our approach, when better data become available, one may reduce the remaining uncertainties considerably and determine the CKM matrix elements with better accuracy. The idea is simple. Both the $B$ and $D$ meson sum rules are determined by one and the same set of nonperturbative parameters, including the coefficients of the nonasymptotic terms in the light-cone wave functions as exemplified in (7). The present uncertainty in these parameters may be further reduced by constraining them through a precise measurement of $D \rightarrow \pi l \nu$. After evolution from $\mu \sim m_{c}$ to $\mu \sim m_{b}$, one should then obtain a more accurate prediction on $B \rightarrow \pi l \nu$ and thus improve also the result on $\left|V_{u b}\right|$.

In conclusion we mention that the second form factor $f_{\pi}^{-}\left(p^{2}\right)$ of the $B \rightarrow \pi$ transition matrix element (2) can be calculated by the same method. The work is in progress [17]. This form factor may become measurable in future experiments in the decay $B \rightarrow \pi \tau \nu_{\tau}$.

\section{The nonfactorizable $B \rightarrow J / \psi K$ amplitude}

To proceed from semileptonic to nonleptonic decays when QCD is switched off, one simply has to replace the lepton current by the relevant quark current in the weak Hamiltonian and multiply the latter by the corresponding CKM matrix element. Of course, the strong interaction effects then become much more complicated. They arise from (a) hard gluon exchange at short distances, (b) soft interactions of quarks and gluons including nonspectator effects, (c) the confinement mechanism leading to the formation of hadrons, and (d) final state interactions among the hadronic decay products.

Theoretically, only the effect (a) can be systematically taken into account in the framework of improved QCD perturbation theory. The result is an effective weak Hamiltonian at the physical scale $\mu<<m_{W}$ of interest, given by a sum of local operators with renormalized Wilson coefficients [18]. In the following we consider the decay $B \rightarrow J / \psi K$ as an important and instructive study case. The part of the effective Hamiltonian relevant for this decay may be written in the form

$$
H_{W}=\frac{G}{\sqrt{2}} V_{c b} V_{c s}^{*}\left\{\left(c_{2}+\frac{c_{1}}{3}\right) O_{2}+2 c_{1} \tilde{O}_{2}\right\}
$$

where

$$
O_{2}(\mu)=\left(\bar{c} \Gamma^{\rho} c\right)\left(\bar{s} \Gamma_{\rho} b\right), \tilde{O}_{2}(\mu)=\left(\bar{c} \Gamma^{\rho} \frac{\lambda^{a}}{2} c\right)\left(\bar{s} \Gamma_{\rho} \frac{\lambda^{a}}{2} b\right)
$$


with $\Gamma_{\rho}=\gamma_{\rho}\left(1-\gamma_{5}\right)$ and $\lambda^{a}$ being the usual SU(3) colour matrices. The Wilson coefficients $c_{i}(\mu)$ contain the QCD corrections from short distances below a characteristic distance scale of the order of the inverse $b$-quark mass. The remaining task is then to calculate the hadronic matrix elements of the four-quark operators (24) incorporating the effects (b) to (d). This clearly requires nonperturbative methods whence progress has been slow and difficult.

In the most radical approach, the matrix elements of $H_{W}$ are factorized into products of hadronic matrix elements of the currents that compose $H_{W}$. Strong interactions at scales lower than $\mu$ between quarks entering different currents as well as nonspectator effects are completely neglected in this approximation. Hence, the resemblance of nonleptonic decays to semileptonic decays goes quite far. Formally, the matrix element of the operator $\tilde{O}_{2}$ vanishes because of colour conservation, and the amplitude for $B(p+q) \rightarrow J / \psi(p) K(q)$ (momenta in parenthesis) is given by the factorized matrix element of the operator $\mathrm{O}_{2}$ :

$$
\left\langle J / \psi K\left|H_{W}\right| B\right\rangle=\frac{G}{\sqrt{2}} V_{c b} V_{c s}^{*}\left(c_{2}(\mu)+\frac{c_{1}(\mu)}{3}\right)\left\langle J / \psi K\left|O_{2}(\mu)\right| B\right\rangle
$$

with

$$
\begin{gathered}
\left\langle J / \psi K\left|O_{2}(\mu)\right| B\right\rangle=\left\langle J / \psi\left|\bar{c} \Gamma^{\rho} c\right| 0\right\rangle\left\langle K\left|\bar{s} \Gamma_{\rho} b\right| B\right\rangle \\
=2 f_{\psi} f_{B \rightarrow K}^{+} m_{\psi}\left(\epsilon^{\psi} \cdot q\right) .
\end{gathered}
$$

While the decay constant $f_{\psi}$ can be obtained from the leptonic width $\Gamma\left(J / \psi \rightarrow l^{+} l^{-}\right)$, the $B \rightarrow K$ form factor $f_{B \rightarrow K}^{+}\left(p^{2}=m_{\psi}^{2}\right)$ can be calculated in analogy to the $B \rightarrow \pi$ form factor discussed in Section 2 . The result used below is $f_{B \rightarrow K}^{+}\left(m_{\psi}^{2}\right)=0.55 \pm 0.05$ 仙. Obviously, $\epsilon^{\psi}$ denotes the $J / \psi$ polarization vector. At this point one encounters a principal problem: since the matrix elements of quark currents in (26) are scale independent, the $\mu$-dependence of $\left\langle J / \psi K\left|O_{2}(\mu)\right| B\right\rangle$ which may cancel the $\mu$-dependence of the Wilson coefficients in (25) and give a physically sensible result, is lost (see also the discussion in [18]). Hence the approximation (26) can at best be valid at a particular value of $\mu$ which could be called the factorization scale $\mu_{F}$. Usually, $\mu_{F}$ is expected to be of the order of the heavy quark mass.

Using the next-to-leading coefficients $c_{1,2}(\mu)$ in the HV scheme for $\Lambda_{\frac{(5)}{M S}}=225 \mathrm{MeV}$ from [19] and taking $\mu=m_{b} \simeq 5 \mathrm{GeV}$, one obtains $a_{2}=0.155$ and from that the branching ratio

$$
B R(B \rightarrow J / \psi K)=0.025 \%
$$

This estimate is significantly smaller than the measured branching ratios [16, 20]:

$$
\begin{gathered}
B R\left(B^{-} \rightarrow J / \psi K^{-}\right)=(0.102 \pm 0.014) \%, \\
B R\left(B^{0} \rightarrow J / \psi \bar{K}^{0}\right)=(0.075 \pm 0.021) \% .
\end{gathered}
$$

This failure, together with the scale problem pointed out above, indicates that factorization of matrix elements has to be accompanied by a reinterpretation of the Wilson coefficients. For class II decays such as $B \rightarrow J / \psi K$, the short-distance $\mu$-dependent coefficient $c_{2}(\mu)+c_{1}(\mu) / 3$ has to be substituted by an effective coefficient $a_{2}$. Phenomenologically [9], the latter is treated as a free parameter to be determined from experiment. From (25), (26), and (28), the most precise of the two measurements, one finds

$$
\left|a_{2}\right|=0.32 \pm 0.02
$$

where the quoted error is purely experimental. The sign of $a_{2}$, of course, remains undetermined. 
More theoretically, one can argue that the above findings are a clear indication for the existence of significant nonfactorizable contributions which account for the difference of $a_{2}$ from the value of $c_{2}+c_{1} / 3$, by roughly a factor of two at $\mu=m_{b}$, and which soften the strong $\mu$-dependence. A deeper analysis shows that the dominant effects should arise from the nonfactorizable matrix element of the operator $\tilde{O}_{2}$. Adopting the parameterization

$$
\left\langle J / \psi K\left|\tilde{O}_{2}(\mu)\right| B\right\rangle=2 \tilde{f}(\mu) f_{\psi} m_{\psi}\left(\epsilon^{\psi} \cdot q\right),
$$

one derives from (23) and (26) the effective coefficient

$$
a_{2}=c_{2}(\mu)+\frac{c_{1}(\mu)}{3}+2 c_{1}(\mu) \frac{\tilde{f}(\mu)}{f_{B \rightarrow K}^{+}} .
$$

We consider the proof of this conjecture and the direct calculation of $a_{2}$ (and of analogous coefficients relevant for the other classes of two-body decays [9]) as one of the most important tasks in heavy flavour physics. As a first step in this direction, we have undertaken a rough estimate of $\tilde{f}$ using again QCD sum rules [21]. Following the general idea put forward in [22], we work with the four-point correlation function

$$
<0\left|T\left\{j_{\mu 5}^{K}(x) j_{\nu}^{\psi}(y) \tilde{O}_{2}(z) j_{5}^{B}(0)\right\}\right| 0>,
$$

where $j_{\mu 5}^{K}=\bar{u} \gamma_{\mu} \gamma_{5} s, j_{\nu}^{\psi}=\bar{c} \gamma_{\nu} c$, and $j_{5}^{B}=\bar{b} i \gamma_{5} u$ are the generating currents of the mesons involved.

On the one side, one considers a dispersion relation for this function in terms of intermediate hadronic states in all three independent kinematical variables (the squared momenta of the currents). Similarly as in (㺼), the ground state contribution contains the matrix element of interest, that is $\left\langle J / \psi K\left|\hat{O}_{2}(\mu)\right| B\right\rangle$. In addition, one has contributions from all kind of excited resonances and continuum states with a very complicated singularity structure.

On the other side, in the deep-euclidean region one can apply a short-distance OPE to the correlation function (33). This leads to a representation by a series of vacuum expectation values (VEV) of local operators with calculable coefficients. The VEV are universal nonperturbative parameters characterizing the QCD vacuum. Best known examples are the gluon and quark condensates. We have performed this calculation including all operators up to dimension 6. More details of our analysis are discussed in [21]. Here we only mention two complications that are not present in the more familiar two- and three-point sum rules. One problem is the presence of a light continuum in the $B$ channel below the $B$-meson pole. This contribution can be associated with processes of the type ' $D^{*} D_{s}$ ' $\rightarrow J / \psi K$ where an initial state carrying $D^{*} D_{s}$ quantum numbers which is created by the combined action of the operator product $\tilde{O}_{2} j_{5}^{B}$ from the vacuum (in (33) ), rescatters into the final $J / \psi K$ state by strong interaction. As a reasonable solution we suggest to cancel this unwanted piece against a corresponding term in the OPE of (33) which develops a nonzero imaginary part at $(p+q)^{2} \geq 4 m_{c}^{2}$ in the channel with the corresponding quark content $c \bar{c} s \bar{q}$. The second problem concerns the subtraction of the contributions from higher resonances and continuum states in the remaining sum rule. Since the normal procedure (mentioned in section 2 ) does not work here, we use a rough model for the spectral function including besides the $B, J / \psi$ and $K$ ground states, only the first excited resonances. We then perform a Borel transformation in the $B$-meson channel and take moments in the charmonium channel. In the $K$-meson channel, we keep the momentum variable $q^{2}$ spacelike. We then fit the spectral function to the sum rule varying 
the Borel mass $M$, the moments and $q^{2}$. From this fit we find $\tilde{f}=-(0.045 \div 0.075)$, where the implicit scale $\mu$ is identified with the central value $M \simeq \sqrt{m_{B}^{2}-m_{b}^{2}} \simeq 2.5 \mathrm{GeV}$. Substituting this estimate in (32), and evaluating the short-distance coefficients $c_{1,2}(\mu)$ also at $\mu=M$ instead of the higher scale $\mu=m_{b}$ used in (27), we obtain

$$
a_{2}=-0.29+0.38-(0.19 \div 0.31)=-(0.10 \div 0.22)
$$

where the three terms refer to the corresponding terms in (32).

Several comments are in order. Firstly, the nonfactorizable matrix element (31) is considerably smaller than the factorizable one given in (26), numerically, $\left|\tilde{f} / f_{B \rightarrow K}^{+}\left(m_{\psi}^{2}\right)\right| \simeq$ 0.1. Nevertheless, it has a strong quantitative impact on $a_{2}$ because of the large coefficient $\left|2 c_{1} /\left(c_{2}+c_{1} / 3\right)\right| \simeq 20 \div 30$. Secondly, the factorizable term $c_{1} / 3$, nonleading in $1 / N_{c}$, and the nonfactorizable term proportional to $\tilde{f}$ are opposite in sign and hence tend to cancel. In fact, if $|\tilde{f}|$ is taken at the upper end of the predicted range, the cancellation is almost complete and as a result increases the branching ratio considerably. This is exactly the scenario anticipated by the $1 / N_{c}$-rule [23 and supported by similar estimates of nonfactorizable amplitudes for $D$-decays [22] and for $B \rightarrow D \pi$ [24, 25]. Thirdly, the theoretical value (34) of $\left|a_{2}\right|$ is still somewhat low as compared to the phenomenological value (30). One should however note that the improvement due to the nonfactorizable contribution is remarkable as the factorizable terms alone would give $\left|a_{2}\right|=0.09$ for $\mu=M$. Finally, our estimate yields a negative overall sign for $a_{2}$ in contradiction to strict factorization (first two terms in (32)) and also to a global fit of the data [20, 26]. We stress, however, that in this fit the positive sign of $a_{2}$ is essentially determined from the channels $B^{-} \rightarrow D^{0} \pi^{-}, D^{0} \rho^{-}, D^{* 0} \pi^{-}$, and $D^{* 0} \rho^{-}$and is then assumed to hold universally for all channels. This assumption may not be correct. Certainly, the theoretical approach described here provides no justification to expect universal values and/or universal signs for the coefficients $a_{i}$ in different channels. From the relation (32) we see that universality can at most be expected within certain classes of decay modes, separately. This point of view is also supported by the phenomenological analysis of nonfactorizable contributions in [27.

As a last remark we emphasize that there is no simple relation between $B$ and $D$ decays in our approach since the OPE for the corresponding correlation functions significantly differ in the relevant diagrams and in the hierarchy of mass scales. We hope to clarify these issues further.

\section{Conclusion}

QCD sum rule techniques are very useful in calculating hadronic matrix elements for exclusive heavy meson decays. In particular, in combination with light-cone wave functions for pions and kaons they provide a powerful tool to derive heavy-to-light form factors and strong couplings of heavy to light mesons. In contrast to other methods including HQET, one can obtain predictions for the whole range in momentum transfer, and also for $D$ mesons which are presumably too light for HQET to work. Moreover, finite mass effects are automatically included.

In this paper, we have focussed on applications to the CKM-suppressed semileptonic decays $B \rightarrow \pi l \nu_{l}$ and $D \rightarrow \pi l \nu_{l}$, and to the factorization approximation of the nonleptonic decay $B \rightarrow J / \psi K$. 
Using the latter mode as a prototype example, we have discussed the shortcomings and principal problems of factorization of nonleptonic amplitudes. Again with the help of QCD sum rule methods, we have directly estimated the nonfactorizable contributions to the amplitude for $B \rightarrow J / \psi K$. Our results indicate that nonfactorizable effects indeed play an essential role, putting the $1 / N_{c}$-rule to work similarly as in $D$-decays. Moreover, on theoretical grounds the nonfactorizable amplitudes are expected to be channel-dependent. Hence, the pattern of twobody weak decays may be much richer than what is revealed by the current phenomenological analysis of the data.

Acknowledgements. R.R. thanks Neville Harnew and Peter Schlein for the opportunity to participate in this fruitful and enjoyable workshop.

\section{References}

[1] M.A. Shifman, A.I. Vainshtein and V.I. Zakharov, Nucl. Phys. B147 (1979) 385, 448.

[2] L.J. Reinders, H.L. Rubinstein and S. Yasaki, Phys. Rep. 127 (1985) 1.

[3] R. Ammar et al. (CLEO Collaboration), Preprint CLEO-Conf 95-09, EPS0165 (1995).

[4] V.M. Belyaev, A. Khodjamirian and R. Rückl, Z. Phys. C60 (1993) 349.

[5] S.J. Brodsky and G.P. Lepage, in: Perturbative Quantum Chromodynamics, ed. A.H. Mueller (World Scientific, Singapore, 1989) pp. 93-240.

[6] V.L. Chernyak and A.R. Zhitnitsky, Phys. Rep. 112 (1984) 173.

[7] V.M. Braun and I.B. Filyanov, Z. Phys. C44 (1989) 157.

[8] V.M. Belyaev, V.M. Braun, A. Khodjamirian and R. Rückl, Phys. Rev. D51 (1995) 6177.

[9] M. Wirbel, B. Stech and M. Bauer, Z. Phys. C29 (1985) 637;

M. Bauer, B. Stech and M. Wirbel, Z. Phys. C34 (1987) 103 .

[10] P. Ball, V.M. Braun and H. Dosch, Phys. Lett. B273 (1991) 316 .

[11] P. Ball, Phys. Rev. D48 (1993) 3190.

[12] S. Barlag et al. (ACCMOR Collab.), Phys. Lett. B278 (1992) 480.

[13] F. Butler et al. (CLEO Collab.), Phys. Rev. Lett. 69 (1992) 2041.

[14] S. Weinzierl, Diploma Thesis, Technical University of Munich, 1994 (unpublished).

[15] G. Rizzo, these proceedings .

[16] Particle Data Group, Phys. Rev. D50 (1994) 1173.

[17] A. Khodjamirian, R. Rückl and C. Winhart, in preparation .

[18] A.J. Buras, these proceedings. 
[19] A.J. Buras, Nucl. Phys. B434 (1995) 606.

[20] M.S. Alam et al. ( CLEO Collab.), Phys. Rev. D50 (1994) 43.

[21] A. Khodjamirian and R. Rückl, in: Continuous Advances in QCD, ed. A.V. Smilga (World Scientific, Singapore, 1994) pp. 287-300, hep-ph/9405310;

A. Khodjamirian, B. Lampe and R. Rückl, in preparation.

[22] B.Yu. Blok and M.A. Shifman, Sov. J. Nucl. Phys. 45 (1987) 135, 301, 522.

[23] A.J. Buras, J.-M. Gerard and R. Rückl, Nucl. Phys. B268 (1986) 16.

[24] B. Blok and M. Shifman, Nucl. Phys. B389 (1993) 534.

[25] I. Halperin, Phys. Lett. B349 (1995) 548.

[26] M. Neubert, V. Rieckert, B. Stech and Q.P. Xu, in : Heavy Flavours, eds. A.J. Buras and M. Lindner ( World Scientific, Singapore, 1992) pp. 286-333.

[27] J. M. Soares, Phys. Rev. D51 (1995) 3518. 